\title{
(6) OPEN ACCESS \\ An international review of the frequency of single-bicycle crashes (SBCs) and their relation to bicycle modal share
}

\author{
Paul Schepers, ${ }^{1}$ Niels Agerholm, ${ }^{2}$ Emmanuelle Amoros, ${ }^{3}$ Rob Benington, ${ }^{4}$ \\ Torkel Bjørnskau, ${ }^{5}$ Stijn Dhondt, ${ }^{6}$ Bas de Geus, ${ }^{6}$ Carmen Hagemeister, ${ }^{7}$ \\ Becky P Y Loo, ${ }^{8}$ Anna Niska ${ }^{9}$
}

${ }^{1}$ Ministry of Infrastructure and the Environment, Delft, The Netherlands

${ }^{2}$ Aalborg University, Aalborg, Denmark

${ }^{3}$ IFSTTAR, Université de Lyon, Lyon, France

'Public Health (Bristol), Bristol, UK

${ }^{5} \mathrm{~T} \varnothing \mathrm{I}$ Institute of Transport Economics, Oslo, Norway ${ }^{6}$ Vrije Universiteit Brussel, Brussels, Belgium

${ }^{7}$ Technische Universität Dresden, Dresden, Germany ${ }^{8}$ University of Hong Kong, Pokfulam, China

${ }^{9} \mathrm{VTI}$, Swedish National Road and Transport Research Institute, Linköping, Sweden

\section{Correspondence to} Paul Schepers, Ministry of Infrastructure and the Environment, PO Box 5044, Delft 2600 GA, Netherlands; paul.schepers@rws.nl

Received 15 July 2013 Revised 26 November 2013 Accepted 15 December 2013 Published Online First 9 January 2014

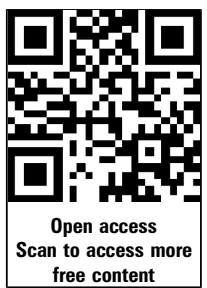

\section{ABSTRACT \\ Objectives To study cyclists' share of transport modes (modal share) and single-bicycle crashes (SBCs) in different countries in order to investigate if the proportion of cyclist injuries resulting from SBCs is affected by variation in modal share.}

Methods A literature search identified figures (largely from western countries) on SBC casualties who are fatally injured, hospitalised or treated at an emergency department. Correlation and regression analyses were used to investigate how bicycle modal share is related to SBCs.

Results On average, $17 \%$ of fatal injuries to cyclists are caused by SBCs. Different countries show a range of values between $5 \%$ and $30 \%$. Between $60 \%$ and $95 \%$ of cyclists admitted to hospitals or treated at emergency departments are victims of SBCs. The proportion of all injured cyclists who are injured in SBCS is unrelated to the share of cycling in the modal split. The share of SBC casualties among the total number of road crash casualties increases proportionally less than the increase in bicycle modal share.

Conclusions While most fatal injuries among cyclists are due to motor vehicle-bicycle crashes, most hospital admissions and emergency department attendances result from SBCs. As found in previous studies of cyclists injured in collisions, this study found that the increase in the number of SBC casualties is proportionally less than the increase in bicycle modal share.

\section{INTRODUCTION}

With increasing attention being paid to the promotion of active, low-carbon travel for health, environmental, social and economic benefits, it is important to understand the relationship between bicycle use and incidence of injury. Single-bicycle crashes (SBCs) are a significant ${ }^{1-3}$ and (in some countries) increasing cause of serious transport related injuries. ${ }^{3-5}$ SBCs cause injuries that result in emergency admission to hospital that are coded as 'non-collision incident' and 'collision with fixed and stationary objects' using the International Classification of Diseases 10. The problem of SBCs has remained hidden for a long time because SBCs are rarely reported in official road crash statistics ${ }^{6-9}$ which do not regularly include hospital data. This may explain why research into SBCs, contributory factors and effects on bicycle use is at an early stage. ${ }^{10}$ It is important to understand SBCs for at least four reasons: (1) SBCs are a significant cause of serious injury, see e.g. figure 1 showing that nonfatal injuries incurred by Dutch cyclists are, regardless of injury severity, mostly due to crashes that do not involve motor vehicles, the large majority being SBCs; (2) SBCs cause direct economic costs through absence from work and from productivity losses; ${ }^{111}$ (3) the hazards that lead to SBCs such as poor infrastructure quality may discourage more active travel by bicycle, thereby preventing people from taking advantage of the health benefits of cycling; ${ }^{12-17}$ and (4) there is a moral obligation to understand the risks of activities that are being promoted so that risks can be minimised or removed and potential participants can grant their informed consent to accept the risks that remain. The causes of SBCs are outside the scope of this paper as these are more fully discussed elsewhere. $^{2} 391018-21$

The relationship between increasing bicycle usage and bicycle crashes involving collisions has received significant research attention, ${ }^{25-27}$ but, as yet, only one Dutch study by Schepers addressed this issue for SBCs. ${ }^{28}$ Schepers found the increase in SBCs to be proportionally less than the increase in bicycle usage, ${ }^{28}$ mirroring the 'non-linearity of risk' found for motor vehicle-bicycle (MV-B) crashes. ${ }^{25} 26$ Most explanations for the nonlinearity of risk relate to MV-B crashes. For instance, Jacobsen ${ }^{25}$ suggests that motorists modify their behaviour when they expect or experience people walking and bicycling, which he called 'safety in numbers'. Different explanations may apply to the risk of SBC: local authorities may improve infrastructure as the amount of cycling increases $^{22}$ and vice versa. ${ }^{29}$ Fewer crashes may occur as cyclists become more experienced and skilled. ${ }^{28}$ To explore whether non-linearity of risk also applies to SBCs, we examined the frequency of SBCs in a sample of countries with varying amounts of cycling.

Using a literature search, we examined (1) the frequency of SBCs resulting in fatal injuries; (2) the frequency of SBCs resulting in severe non-fatal injuries (here defined as casualties who are admitted to the hospital (hospitalised) or treated at an emergency department (ED)); (3) how bicycle modal share is associated with the proportion of cycling injuries occurring in SBCs; and (4) the hypothesis that an increase in bicycle modal share is related to a less than proportional increase in the share of SBCs among all road crash victims. We aimed to include data from countries with varying 


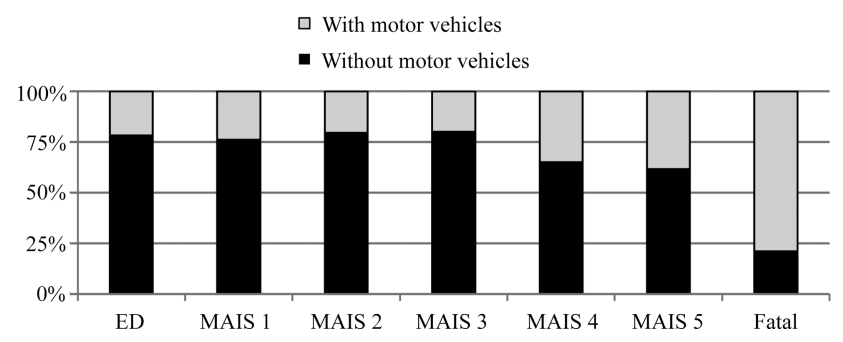

Figure 1 Distribution of injuries incurred by cyclists in crashes with and without motor vehicles in the Netherlands (ED, emergency department). ${ }^{22-24}$

bicycle modal shares to allow for hypothesis testing. We did not restrict the study to specific countries, but most published research is from Western countries.

\section{METHODS}

\section{Data sources}

We searched for SBC and modal share figures from countries with varying bicycle modal shares. In this study, SBCs are defined as falls and obstacle collisions (all crash types in which only the cyclist is involved). Other typical bicycle crash types, not included in this study, are collisions with motor vehicles, cyclists and pedestrians. We used Google Scholar, SafetyLit and Scopus to search for studies about SBCs with 'single-bicycle crash' and 'bicycle-only crash' as search terms. We contacted research organisations for grey literature and included this alongside published studies. In some cases, we also contacted authors of the publications for additional information. We also searched for literature in which bicycle modal shares are reported to allow for hypothesis testing. The data sources are further described for fatal injuries, hospitalised injuries and injuries treated at an ED. This also includes data on modal share that are used to study the relationship with SBCs.

\section{Fatalities}

SBCs are rarely reported by the police, but crashes with a higher injury severity, especially fatal crashes, are associated with an increased reporting and recording rate. ${ }^{7} 830$ Therefore, we used existing reviews by ETSC $^{31}$ and IRTAD $^{32}$ which included police reported data on fatalities in European countries. Other data sources for fatalities in SBCs are not sufficiently available as yet. We restricted analyses on fatalities to Europe because a study by Gallup $^{33}$ identified modal share in European countries using the same method for each country. Per country 1000 people answered the following question: 'What is the main mode of transport that you use for your daily activities?'33

\section{Hospitalised injuries}

The search identified 13 studies that included figures for hospitalised casualties from hospital records or questionnaires filled out by hospitalised victims. Studies focused on a smaller area such as a city were excluded in case we found a study for a whole county. Also, if there were two sources for the same area, we included the most recent one only. For purposes of comparability, the categories 'unknown' or 'unspecified' (in the studies used for this investigation) are excluded from the estimated proportions of SBC victims. Some studies only reported the share of injuries due to bicycle crashes with no motor vehicles, which also includes bicycle-bicycle and bicycle-pedestrian crashes. Based on other studies, it was estimated that $90 \%$ were a victim of a SBC. ${ }^{18-202334}$ For seven out of 13 study areas, we could use the study by Gallup to determine bicycle modal share. For five non-European countries, modal share data were obtained from other sources (such as Pucher and Buehler ${ }^{35}$ for Canada, USA and Australia) which are based on National Travel Surveys (NTS). Bicycle modal share in such studies is determined using travel diaries. The data from Gallup and NTSs may vary, which is further discussed in the Analyses section.

\section{Injuries treated at an ED}

The search identified 13 studies including figures for casualties treated at an ED. The same protocol as for hospital admissions (see Hospitalised injuries section) was followed for selection of studies and data processing. As we could not find data on bicycle modal share for a sufficiently large number of study areas, hypothesis testing is restricted to fatal and hospitalised injuries and excludes ED treatments.

\section{Analyses}

A Pearson correlation is calculated between the share of SBCs and bicycle modal share to examine this relationship. However,

Table 1 Proportion of SBC casualties among total number of bicycle crash and road crash casualties admitted to hospital per country

\begin{tabular}{|c|c|c|c|c|}
\hline \multirow{2}{*}{$\begin{array}{l}\text { Study area, study } \\
\text { period and sources }\end{array}$} & \multirow{2}{*}{$\begin{array}{l}\text { Share of cycling } \\
\text { in the modal split }\end{array}$} & \multicolumn{2}{|c|}{ Share (\%) of SBC victims among } & \multirow{2}{*}{$\begin{array}{l}\text { Number of cyclist } \\
\text { victims on which the share is based }\end{array}$} \\
\hline & & Cyclists & All road crash injuries & \\
\hline Netherlands $(2005-2009)^{4} 33$ & 31 & 74 & 41 & 46100 \\
\hline Denmark $(2011)^{33} 36$ & 19 & 74 & 33 & 18489 \\
\hline Belgium, Flanders and Brussels (2003-2007) ${ }^{133}$ & 13 & 87 & 30 & 18750 \\
\hline England $(2011-2012)^{1933}$ & 2 & 80 & 23 & 16227 \\
\hline Sweden $(1998-2005)^{5} 33$ & 17 & 75 & 23 & 29788 \\
\hline Finland $(1985-1986)^{* 33} 37$ & 13 & 65 & 22 & 127 \\
\hline Canada $(2010-2011)^{35} 38$ & 2 & 82 & 15 & 4126 \\
\hline USA, Oregon (2002-2006) 353940 & 1 & 67 & 11 & 414 \\
\hline Australia $(2008-2009)^{34} 35$ & 1 & 63 & 9 & 3811 \\
\hline France, Rhône county (1996-2008) ${ }^{20} 33$ & 3 & 65 & 9 & 2578 \\
\hline $\operatorname{Iran}(2003)^{41}$ & NA & 62 & 3 & 420 \\
\hline Germany, Münster (2009-2010*9 35 & 27 & 72 & & 251 \\
\hline New Zealand $(2011)^{42} 43$ & 1 & 78 & & 2260 \\
\hline
\end{tabular}

${ }^{*}$ Questionnaire study among hospitalised victims.

SBC, single-bicycle crash. 
Table 2 Proportion of SBC casualties in the total number of cyclist crash casualties treated at emergency departments per country

\begin{tabular}{|c|c|c|}
\hline Study area, study period and sources & Share (\%) of SBC victims & $\begin{array}{l}\text { Number of cyclist victims } \\
\text { on which the share is based }\end{array}$ \\
\hline Netherlands $(2005-2009)^{23}$ & 78 & 6830 \\
\hline England, Cambridge $(2003)^{\star 44}$ & 69 & 293 \\
\hline Germany, Göttingen $(2007-2008)^{* 45}$ & 60 & 294 \\
\hline Sweden $(2007-2011)^{18}$ & 77 & 37563 \\
\hline Finland $(1985-1986)^{* 37}$ & 79 & 260 \\
\hline Norway $(2001-2002)^{46}$ & 82 & 991 \\
\hline France, Rhône county (1996-2008) ${ }^{20}$ & 71 & 13684 \\
\hline Austria, leisure cycling $(2007-2009)^{* 47}$ & 80 & 512 \\
\hline USA, California, New York and North Carolina $(1993-1997)^{48}$ & 63 & 2509 \\
\hline Canada $(2006)^{49}$ & 89 & 3817 \\
\hline Hong Kong, Shatin, New Territories $(2006)^{50}$ & 88 & 1315 \\
\hline Turkey, Central Anatolian Region (2005-2008) $)^{51}$ & 95 & 150 \\
\hline United Arab Emirates $(2001-2003)^{52}$ & 84 & 200 \\
\hline
\end{tabular}

*Questionnaire study among victims treated at an emergency department.

SBC, single-bicycle crash.

the bicycle modal share measure used to correlate with hospitalised casualties contains data from both the Gallup study and from NTS studies. To control for possible differences we have created a control variable that equals zero for Gallup based measures and one for NTS based, that is, a dummy variable. Linear regression analysis is conducted on the share of hospitalised SBC casualties in the total number of hospitalised casualties with bicycle modal share and the control variable for bicycle modal share measurement method as independent variables.

To investigate whether an increase in bicycle modal share is related to a less than proportional increase in the share of SBCs among all road crash victims, trend lines of the form SSBCT $=\alpha$ $\mathrm{BMS}^{\beta}$ (ie, a power function) have been fitted to the data using SPSS. SSBCT is the Share of SBC casualties in the Total number of road traffic casualties while BMS stands for Bicycle Modal Share. Exponent $\beta$ indicates the change in SSBCT in response to changes in BMS. With $\beta$ equal to 1 , the growth in SSBCT with increasing BMS would be linear; $\beta$ less than 1 indicates the growth in crashes would be less than linear (which would support our hypothesis). Also, the dummy variable for bicycle modal share measurement method is included as a control in these analyses.

Again, for hospitalised casualties the control variable is added to the equation to address bicycle modal share measurement method: SSBCT $=\alpha \mathrm{BMS}^{\beta 1} \operatorname{Exp}(\beta 2$ Dummy). Exponent $\beta 2$ indicates the effect of measurement method. It has no effect if $\beta 2$ equals $0(\operatorname{Exp}(0)=1)$.

\section{RESULTS}

This section reports our findings on the incidence of SBC injuries (see Fatalities and Hospitalisations and ED treatments sections) and whether the proportion of SBC casualties is associated with changes in cycling's modal share (see Is bicycle modal share associated with the proportion of cycling injuries occurring in SBCs? section).

\section{Fatalities}

Data on fatalities are derived from police recorded crash statistics. On average in European countries, $17 \%$ of cyclist fatalities are killed by SBCs, but the proportion for different countries varies between $5 \%$ and $30 \% .^{31}$ As a proportion of the total number of traffic fatalities (including car occupants, motorcyclists, etc), the share ranges between a few hundredths of a percent and $4 \% .^{31} 32$ The large variation may result from relatively low number of cyclist fatalities in countries with low amounts of cycling.

\section{Hospitalisations and ED treatments}

Tables 1 and 2 show the proportion of SBC casualties among all injured cyclists admitted to hospitals or treated at EDs. Data are derived directly from medical registrations in most studies, but some are questionnaire studies among patients. The studies vary in size: some studies based on hospital data apply to the whole population in an area. Studies based on samples tend to be smaller, focused on data provided by one or several hospitals. The sample of injured cyclists on which the proportion of SBCs is based is included as an indication of study size. The results reveal that the share of SBC victims varies between $60 \%$ and $95 \%$ of all cyclist casualties, admitted to hospital or treated at an ED. As a proportion of the total number of hospitalised traffic casualties (including car occupants, motorcyclists, etc), the share exceeds $20 \%$ in six out of 11 countries for which this proportion is known.

\section{Is bicycle modal share associated with the proportion of cycling injuries occurring in SBCs?}

This section describes the relationship between the proportion of cyclist casualties (both fatalities and hospitalisations) injured in SBCs and the share of cycling in the modal split. Table 3 shows descriptive statistics and correlation coefficients. The Pearson correlation coefficients for the relationship between the share of cycling in the modal split and the share of SBC casualties among cyclist casualties are small and not significant, indicating that the variables are unrelated. The results show that the proportion of all journeys made by bicycle is not related to the proportion of cycling injuries occurring in SBCs.

The correlation between the share of cycling in the modal split and the share of SBC casualties in the total number of road traffic casualties is significant. The higher the proportion of cycle journeys in the modal split, the higher the percentage of all road traffic injuries caused by SBCs. This is to be expected: more cyclist casualties can be expected where there are more cyclists. The high correlation also supports the validity of the bicycle modal share measures used for this study. 
Table 3 Descriptive statistics and the relationship between the share of SBC casualties and the share of cycling in the modal split (shares measured in percentages)

\begin{tabular}{|c|c|c|c|c|c|c|c|c|c|}
\hline & \multirow{2}{*}{ Mean } & \multirow{2}{*}{ SD } & \multirow{2}{*}{$\mathrm{Nt}$} & \multicolumn{6}{|c|}{ Pearson correlation } \\
\hline & & & & 1 & 2 & 3 & 4 & 5 & 6 \\
\hline \multicolumn{10}{|l|}{ Fatalities in European countries (2008-2010): } \\
\hline 1. Share of cycling in the modal split ${ }^{33}$ & 10 & 8 & 19 & 1 & 0.01 & $0.56^{*}$ & & & \\
\hline 2. Share of SBC fatalities in the total number of cyclist fatalities ${ }^{31}$ & 17 & 9 & 20 & & 1 & $0.81^{* *}$ & & & \\
\hline 3. Share of SBC fatalities in the total number of road traffic fatalities ${ }^{31} 32$ & 2 & 1 & 18 & & & 1 & & & \\
\hline \multicolumn{10}{|l|}{ Hospitalised casualties (from table 1 ) } \\
\hline 4. Share of cycling in the modal split & 11 & 11 & 12 & & & & 1 & 0.09 & $0.90^{* *}$ \\
\hline 5. Share of SBC casualties in the total number of cyclist casualties & 73 & 8 & 13 & & & & & 1 & 0.59 \\
\hline 6. Share of SBC casualties in the total number of hospitalised road traffic casualties & 20 & 12 & 11 & & & & & & 1 \\
\hline
\end{tabular}

Linear regression analysis on the share of hospitalised SBC casualties in the total number of hospitalised casualties has been conducted and includes a control variable for bicycle modal share measurement method to check whether the measurement method affects the result. Table 4 shows the results. The correlation of 0.09 between bicycle modal share and the proportion of hospitalised SBC casualties in the total number of hospitalised cyclist casualties reduces to 0.05 after correcting for measurement method. This supports the result that measurement method did not affect the result significantly and that the proportion of all journeys made by bicycle is not related to the proportion of cycling injuries occurring in SBCs; the proportion remains constant although within a wide range of between $60 \%$ and $95 \%$.

\section{How is bicycle modal share related to the proportion of all road traffic injuries that occur in SBCs?}

This section explores the hypothesis that an increase in bicycle modal share is related to a less than proportional increase in the share of SBCs among all road crash victims. Table 5 shows the results of the analyses of the proportion of SBC casualties in the total number of road traffic casualties. In line with the hypothesis, the exponent for the growth in the share of both SBC fatalities and hospitalised SBC casualties is smaller than 1, but only the parameter for hospitalised casualties is significantly lower than 1 . The latter may be due to the relatively low fatality numbers, which increases the SD. The results suggest that the higher the proportion of all journeys made by bicycle, the lower the increase in the proportion of cyclist injuries caused by SBCs will be. The coefficient for the

Table 4 Estimation results for regression analysis on the share of hospitalised SBC casualties in the total number of hospitalised casualties (95\% Wald Cl)

\begin{tabular}{lcl}
\hline & Regression coefficient & $\begin{array}{l}\text { Standardised } \\
\text { regression } \\
\text { coefficient }\end{array}$ \\
\hline $\begin{array}{l}\text { Constant } \\
\text { Share of cycling in the modal }\end{array}$ & $0.04(-0.52$ to 0.59$)$ & - \\
$\begin{array}{l}\text { split } \\
\text { NTS versus Gallup (dummy }\end{array}$ & $-1.62(-13.28$ to 10.04) & -0.11 \\
$\begin{array}{l}\text { variable) } \\
\text { Model fit, adjusted R }\end{array}$ & $2 \%$ & \\
\hline NTS, National Travel Surveys; SBC, single-bicycle crash. &
\end{tabular}

control variable for measurement method is close to zero and has hardly any effect.

\section{DISCUSSION}

Between $60 \%$ and $95 \%$ of cyclists admitted to hospitals or treated at EDs are victims of SBCs showing the significance of this problem. As a proportion of the total number of hospitalised traffic casualties, the share averages at $20 \%$ which is about twice as high as the average bicycle modal share. The high number of cyclists injured in SBCs cause a significant health burden both directly (through the health and social care costs of injury and economic costs including absence from work and productivity losses) and indirectly (by discouraging more active and sustainable travel choices). The size of the problem warrants more research to inform and improve preventative measures. Moreover, this study shows the importance of using medical data alongside police data to achieve a more complete picture of the cycling safety issue as regards injuries admitted to hospital or treated at EDs.

In support of the hypothesis, we found that the increase in the share of SBC casualties in the total number of road crash casualties is proportionally less than the increase in the share of cycling in the modal split; increasing modal share does not appear to increase the SBC injury burden at the same rate. But this result was only statistically significant for hospitalised casualties, not for fatalities.

The proportion of cyclist casualties killed in or hospitalised due to SBCs is not found to be related to the share of cycling in the modal split. However, this probably only holds for currently

Table 5 Estimation results for regression analyses on the share of SBC casualties in the total number of road traffic casualties $(95 \%$ Wald $\mathrm{Cl}$ )

\begin{tabular}{|c|c|c|}
\hline & Fatalities & $\begin{array}{l}\text { Hospitalised } \\
\text { casualties }\end{array}$ \\
\hline Constant & 0.24 (0.10 to 0.56$)$ & 10.19 (5.04 to 20.63$)$ \\
\hline $\begin{array}{l}\text { Exponent for growth in bicycle } \\
\text { modal share }\end{array}$ & $0.71(0.33$ to 1.10$)$ & 0.37 (0.09 to 0.65$)$ \\
\hline $\begin{array}{l}\text { NTS versus Gallup (dummy } \\
\text { variable) }\end{array}$ & - & $0.03(-0.74$ to 0.80$)$ \\
\hline Model fit, $\mathrm{R}^{2}$ & $51 \%$ & $75 \%$ \\
\hline
\end{tabular}

NTS, National Travel Surveys; SBC, single-bicycle crash. 
observed modal shares and not for higher ones. It seems unlikely that were cycles to be used for almost $100 \%$ of journeys the injurious collisions with other cycles and pedestrians would increase sufficiently to replace the numbers of MV-B crashes, because the former are generally less serious than the latter. The authors suppose that there is a threshold in modal share of cyclists up to which increases do not result in significant increases in the proportion of cyclist casualties resulting from SBCs. Above this level, further increases in cycling as a share of all journeys will increase incidence of SBCs as a proportion of all cycle injuries. Further research into this phenomenon is required and, until then, the proven dissociation between modal share and SBC as a proportion of all injures needs to be qualified, because it may apply only to the modal share proportions of between $1 \%$ and $31 \%$ identified by and included in this report. Note that this line of reasoning is only relevant to shares of crash types and not to changes in absolute numbers of road crash casualties which is further discussed by others. ${ }^{22} 26$

Other studies have shown that the number of cyclist casualties in collisions (per passing cyclist or per kilometre cycled) reduces as the amount of cycling increases. ${ }^{22} 25{ }^{27}$ For instance, according to Jacobsen, taking into account the amount of cycling, the probability that a motorist will collide with a cyclist declines with the roughly -0.6 power of the volume of cyclists. ${ }^{25}$ This phenomenon -that the increase in injury is not directly proportional to the increase in the number of cyclists on the road-has been called the non-linearity of risk. ${ }^{26}$ The relatively constant distribution between SBCs and other crash types irrespective of the modal split suggests that the non-linearity of risk applies to all bicycle crash types including SBCs. To illustrate this, we may suppose the nonlinearity only applies to MV-B crashes. In this case, the occurrence of $M V-B$ crashes would decrease with increasing shares of cycling in the modal split, while the number of SBCs would remain constant. The result would be a decrease in the share of $M V-B$ crashes and an increase in the share of SBCs among cycling injuries as modal share of cycling increases. Table 3 presents insignificant correlations $(0.01$ for fatalities; 0.09 for hospitalised casualties) showing that the latter is not the case. This result is in accordance with earlier research suggesting a non-linear relationship between risk and exposure for SBCs and suggests that this also applies to fatal SBCs. ${ }^{28}$

Since safety in numbers cannot offer an explanation of the non-linearity of risk of injuries that do not involve motor vehicles, what factors might explain this phenomenon? We discuss explanations that can be studied in future research. Infrastructure may be improved in response to demand from increasing numbers of cyclists, and vice versa. ${ }^{27} 53$ For instance, good (winter) maintenance and removing or improving the visibility of obstacles can help reduce the risk of SBCs and improve conditions for cyclists, especially those with poor vision. On the contrary, in the USA where cycling enjoys a very low modal share some cities have designated sidewalks as bicycle paths, where cyclists are confronted with fixed objects such as parking metres, utility poles, signposts and trees. ${ }^{54}$ We cannot yet prove a causal relationship among level of cycling, quality of cycle infrastructure and incidence of SBCs, but a relationship is conceivable given the high standards regarding these aspects in countries with high amounts of cycling. A more structured comparison of infrastructure quality between countries with varying bicycle modal shares could yield valuable data to explain the non-linearity of risk and help find ways to reduce risk. We recommend investing in infrastructure that improves both cycling safety and the attractiveness of cycling, building on the growing understanding of the causes of SBCs which are presented elsewhere. ${ }^{2} 31018-21$
Another line of reasoning to explain the non-linearity of risk follows from the assumption that inexperienced and cautious cyclists are at a lower risk of SBCs injury. Consistent with differences in risk aversion, female commuter cyclists and children (especially girls) prefer routes with maximum separation from motorised traffic. ${ }^{55-58}$ Especially these groups cycle more in countries with high amounts of cycling. ${ }^{1135}$ However, the elderly also run a higher risk because they are most vulnerable. ${ }^{28}$ It requires more research to examine whether differences in the population of cyclists between countries with varying bicycle modal shares explain part of the non-linearity of the risk of SBCs; see for example, Fyhri $e t a l^{59}$ for groups with different risk profiles in the population of cyclists. A design as used in this study would help, but would need to be expanded with much more detailed information on crashes and bicycle usage in age groups, gender classes, income classes and so on. Instead of the modal split variable used in this study which was derived from a survey question about people's main mode of transport for daily activities, such research requires more detailed data from NTS.

Another type of research that we would like to recommend is the development and use of alternative data sources for fatally injured SBC casualties. We used police reported data for fatalities. Even though the most severe casualties are more likely to be recorded by the police, we suspect that our outcome may be an underestimate. Data from such sources are rarely reported in scientific literature, but could yield a more reliable estimate of the share of SBCs among road traffic fatalities. Regarding hospital data, we recommend research on the quality of road crash classification in medical registrations. The International Classification of Diseases is of great value for international comparisons such as this study, but it could be that reporting of certain crash types is correlated to their frequency. Data quality may be improved by restricting to a higher level of injury severity instead of hospitalisations, for example, an MAIS of 3 or more about which data may become more widely available for European countries in the future. ${ }^{60}$

\section{What is already known on the subject}

- Only a small proportion of single-bicycle crashes are recorded in police statistics, but other sources of information about their causes exist.

- More cyclists are hospitalised due to single-bicycle crashes than as a result of collisions with motorists-the reverse holds for fatalities.

- Cyclists are less likely to be involved in collisions with motor vehicles as the numbers of cyclists and amount of cycling increase.

\section{What this study adds}

Between $60 \%$ and $95 \%$ of cyclists admitted to hospitals or treated at emergency departments are single-bicycle crash victims.

- The proportions of cycling fatalities and hospital admissions due to single-bicycle crashes are not found to be related to bicycle modal share.

- However, increasing bicycle modal share is associated with a reduction in the proportion of all road traffic injuries caused by single-bicycle crashes. 
Acknowledgements The authors wish to thank the researchers and institutions who were willing to provide data.

Contributors All authors have substantially contributed to the conception and design of the study, interpretation of the analysed data, and preparation of the paper.

\section{Competing interests None.}

Provenance and peer review Not commissioned; externally peer reviewed.

Data sharing statement Data used in this study are derived from a literature review, that is, there are no unpublished data in the study.

Open Access This is an Open Access article distributed in accordance with the Creative Commons Attribution Non Commercial (CC BY-NC 3.0) license, which permits others to distribute, remix, adapt, build upon this work non-commercially, and license their derivative works on different terms, provided the original work is properly cited and the use is non-commercial. See: http://creativecommons.org/ licenses/by-nc/3.0/

\section{REFERENCES}

1 Dhondt S, Macharis C, Terryn N, et al. Health burden of road traffic accidents, an analysis of clinical data on disability and mortality exposure rates in Flanders and Brussels. Accid Anal Prev 2013;50:659-66.

2 Nyberg P, Björnstig U, Bygren LO. Road characteristics and bicycle accidents. Scand J Soc Med 1996;24:293-301.

3 Boufous $\mathrm{S}$, de Rome L, Senserrick T, et al. Single-versus multi-vehicle bicycle road crashes in Victoria, Australia. Inj Prev 2013;19:358-62.

4 Reurings MCB, Bos NM. Ernstig verkeersgewonden in de periode 1993-2009 (Serious road injuries in the period 1993-2009). Leidschendam: Institute for Road Safety Research, 2011.

5 Larsson J. Trafikskador 1998-2005 enligt patientstatistik (Traffic injuries 19982005, according to patient statistics). Linköping: VTI, Swedish National Road and Transport Research Institute, 2008.

6 Langley JD, Dow N, Stephenson S, et al. Missing cyclists. Inj Prev 2003;9:376-9

7 Loo BPY, Tsui KL. Factors affecting the likelihood of reporting road crashes resulting in medical treatment to the police. Inj Prev 2007;13:186-9.

8 Veisten K, Sælensminde K, Alvær K, et al. Total costs of bicycle injuries in Norway: Correcting injury figures and indicating data needs. Accid Anal Prev 2007;39:1162-9.

9 Juhra C, Wieskotter B, Chu K, et al. Bicycle accidents-do we only see the tip of the iceberg? A prospective multi-centre study in a large German city combining medical and police data. Injury 2012;43:2026-34.

10 Schepers JP, den Brinker BPLM. What do cyclists need to see to avoid single-bicycle crashes? Ergonomics 2011;54:315-27.

11 de Geus $B$, Vandenbulcke $G$, Int Panis $L$, et al. A prospective cohort study on minor accidents involving commuter cyclists in Belgium. Accid Anal Prev 2012;45:683-93.

12 de Geus B, van Hoof E, Aerts I, et al. Cycling to work: influence on indexes of health in untrained men and women in Flanders. Coronary heart disease and quality of life. Scand J Med Sci Sports 2008;18:498-510.

13 Oja P, Titze S, Bauman A, et al. Health benefits of cycling: a systematic review. Scand J Med Sci Sports 2011:21:496-509.

14 Bassett DR, Pucher J, Buehler R, et al. Walking, cycling, and obesity rates in Europe, North America, and Australia. J Phys Act Health 2008;5:795-814.

15 Bergström A, Magnusson R. Potential of transferring car trips to bicycle during winter. Transportation Res Part A 2003;37:649-66.

16 Fabriek E, De Waard D, Schepers JP. Improving the visibility of bicycle infrastructure Int J Hum Factors Ergon 2012;1:98-115.

17 Ormel W, Klein Wolt K, Den Hertog P. Enkelvoudige fietsongevallen (Single-bicycle crashes). Amsterdam: Stichting Consument en Veiligheid, 2008.

18 Niska A, Gustafsson S, Nyberg J, et al. Cyklisters singelolyckor Analys av olycks- och skadedata samt djupintervjuer. (Single bicycle accidents. Analysis of hospital injury data and interviews). Linköping: VTI, Swedish National Road and Transport Research Institute, 2013.

19 Benington R. An introduction to non-collision cycling incidents. Bristol: NHS, 2012. http://www.tinyurl.com/avonsafecycling

20 Amoros $E$, Chiron $M$, Thélot $B$, et al. The injury epidemiology of cyclists based on a road trauma registry. BMC Public Health 2011;11:653.

21 Hagemeister C, Tegen-Klebingat A. Fahrgewohnheiten älterer Radfahrerinnen und Radfahrer. Köln: TÜV Media, 2011.

22 Schepers JP, Heinen E. How does a modal shift from short car trips to cycling affect road safety? Accid Anal Prev 2013:50:1118-27.

23 Consumer Safety Institute. Factsheet fietsongevallen (Factsheet Bicycle Victims). Amsterdam: Stichting Consument en Veiligheid, 2011.

24 SWOV. Cognos. https://www.swov.nl/ibmcognos/cgi-bin/cognos.cgi? (accessed 23 Mar 2013)
25 Jacobsen PL. Safety in numbers: more walkers and bicyclists, safer walking and bicycling. Inj Prev 2003;9:205-9.

26 Elvik R. The non-linearity of risk and the promotion of environmentally sustainable transport. Accid Anal Prev 2009:41:849-55.

27 Robinson DL. Safety in numbers in Australia: more walkers and bicyclists, safer walking and bicycling. Health Promot J Austr 2005;16:47-51.

28 Schepers JP. Does more cycling also reduce the risk of single-bicycle crashes? Inj Prev 2012:18:240-5.

29 Dill J, Carr T. Bicycle commuting and facilities in major U.S. cities: if you build them commuters will use them-another look. Transp Res Rec 2003;1828:116-23.

30 Amoros $\mathrm{E}$, Martin JL, Lafont $\mathrm{S}$, et al. Actual incidences of road casualties, and their injury severity, modelled from police and hospital data, France. Eur J Public Health 2008:18:360-5.

31 ETSC. Pedalling towards Safety. Brussels: European Transport Safety Counsil, 2012.

32 IRTAD. http://www.irtad.net (accessed 3 Jul 2013)

33 Gallup. Flash Eurobarometer "Future of transport" (No 312): European Commission: Directorate General Mobility and Transport, 2011.

34 Henley G, Harrison JE. Serious injury due to land transport accidents, Australia 2008-09. Canberra: Australian Institute of Health and Welfare, 2012.

35 Pucher J, Buehler R. Making cycling irresistible: lessons from the Netherlands, Denmark and Germany. Transport Rev 2008;28:495-528.

36 Statistics Denmark. Traffic Accidents. http://www.dst.dk/ (accessed 31 Jan 2013).

37 Olkkonen S, Lahdenranta U, Slätis $\mathrm{P}$, et al. Bicycle accidents often cause disability-an analysis of medical and social consequences of nonfatal bicycle accidents. Scand J Soc Med 1993;21:98-106.

38 CIHI. The National Trauma Registry (NTR). Ottawa: Canadian Institute for Health Information, 2012

39 Purcell H. An overview of non-motor vehicle bicycle crashes in Oregon. WREN Conference. Ashland, Oregon, 2008.

40 Oregon Health Authority. Oregon injury prevention plan 2011-2015. Salem, Oregon: Oregon Health Authority, Division of Public Health, 2010.

41 Karkhaneh $\mathrm{M}$, Naghavi $\mathrm{M}$, Rowe BH, et al. Epidemiology of bicycle injuries in 13 health divisions, Islamic Republic of Iran 2003. Accid Anal Prev 2008; 40:192-9.

42 Ministry of Transport. Cyclists; crash factsheet 2012. Wellington: Ministry of Transport, 2012

43 Ministry of Transport. Raising the profile of walking and cycling in New Zealand. Wellington: Ministry of Transport, 2008.

44 Davidson JA. Epidemiology and outcome of bicycle injuries presenting to an emergency department in the United Kingdom. Eur J Emerg Med 2005; $12: 24-9$.

45 Ellwein A. Verletzungsmuster und Verletzungsschwere bei Fahrradunfällen im Großraum Göttingen. Göttingen: Georg-August-Universität, 2011.

46 Hansen KS, Engesaeter LB, Viste A. Protective effect of different types of bicycle helmets. Traffic Inj Prev 2003;4:285-90.

47 KfV. IDB Austria. Vienna: Kuratorium für Verkehrssicherheit, 2010.

48 Stutts JC, Hunter WW. Injuries to pedestrians and bicyclists: an analysis based on hospital emergency department data. Chapel Hill: University of North Carolina, 1999.

49 PHAC. Injuries associated with bicycles: Canadian Hospitals Injury Reporting and Prevention Program (CHIRPP). Ottawa, Ontario: Public Health Agency of Canada, 2006.

50 Yeung $\mathrm{JH}$, Leung CS, Poon WS, et al. Bicycle related injuries presenting to a trauma centre in Hong Kong. Injury 2009;40:555-9.

51 Özkan S, Akdur O, İkizceli İ, et al. Bicycle related injuries in adults and children in the central Anatolian region: analysis of 4 years. J Acad Emerg Med 2012;11:35-40.

52 Eid HO, Bashir MM, Muhammed OQ, et al. Bicycle-related injuries: a prospective study of 200 patients. Singapore Med J 2007:48:884-6.

53 Schepers JP, Hagenzieker MP, Methorst R, et al. A conceptual framework for road safety and mobility applied to cycling safety. Accid Anal Prev 2013.In press.

54 Wachtel A, Lewiston D. Risk factors for bicycle-motor vehicle collisions at intersections. ITE J 1994:64:30-5.

55 Gerrard J, Rose G, Lo SK. Promoting transportation cycling for women: the role of bicycle infrastructure. Prev Med 2008;46:55-9.

56 Lord A, Murray S. Cycling-A secondary issue: cycling in leeds secondary schools years 7, 8 and 9. Leeds: Leeds City Council, 2004.

57 Stinson MA, Bhat CR. A comparison of the route preferences of experienced and inexperienced bicycle commuters. Transportation Research Board, 2005.

58 Bernhoft IM, Carstensen G. Preferences and behaviour of pedestrians and cyclists by age and gender. Transportation Res Part F 2008;11:83-95.

59 Fyhri A, Bjørnskau T, Backer-Grøndahl A. Bicycle helmets-A case of risk compensation? Transportation Res Part F: Traffic Psychology and Behaviour 2012;15:612-24.

60 Breen J. High-level group on road safety consultation on the development of the injuries strategy. Skipton, 2012. 